\title{
A histochemical study of cadmium binding with frog cardiac muscle fiber
}

\author{
Hideo Hayashi* and Etsuko Horiuchi** \\ Department of Physiology, Nippon Medical School
}

Chemical analysis on the cadmium content in various organs disclosed that trace of cadmium was found even in the hearts of some healthy animals and $\mathrm{men}^{1,2)}$. As a large amount of cadmium was found in the heart of patient of chronic cadmium intoxication ${ }^{3}$, cadmium is considered to have high affinity to some structural components of the hearts. On the other hand, it has been suggested from the authors' electrophysiological experiments on the contractility of the frog heart that $\mathrm{Cd}$ ions may competitively occupy the marginal zone of the cardiac muscle fiber where $\mathrm{Ca}$ ions normally exist ${ }^{4}$. The purpose of the present paper is to demonstrate histochemically the binding of $\mathrm{Cd}$ with the frog cardiac muscle fiber.

The hearts of bull frogs (Rana catesbeiana) were excised out immediately after intrathecal urethane anethesia (1g per kg body weight). The hearts were divided into two or four strips along the heart axes. One halves of the hearts were soaked into Ringer's solution $\left(\mathrm{NaCl} 110 \mathrm{mM}, \mathrm{KCl} 2 \mathrm{mM}, \mathrm{CaCl}_{2} 1 \mathrm{mM}\right.$, glucose $10 \mathrm{mM}$, tris buffer $\left.10 \mathrm{mM}, \mathrm{pH} 7.2\right)$ containing various concentrations of $\mathrm{Cd}$ and the other halves of the hearts were soaked into Ringer's solution without $\mathrm{Cd}$ as control.

\section{Macroscopic observation}

Both halves of the frog hearts pretreated with and without $\mathrm{Cd}$ were soaked in a $\mathrm{pH} 4.0$ Ringer's solution (phthalate buffer) saturated with $\mathrm{H}_{2} \mathrm{~S}$ for 10 minutes and were fixed in pure ethanol for more than 10 minutes. $\mathrm{H}_{2} \mathrm{~S}$ reacted with $\mathrm{Cd}$ and yellow cadmium sulfide precipitates were formed in the tissue. Fig. 1 illustrates the macroscopic color difference between the control half (right) and $\mathrm{Cd}$ pretreated half (left) : the $\mathrm{Cd}$ pretreated heart (Fig. 1-A) was more yellowish than the control (Fig. 1-B) distinctively. The color difference of staining between $0.1 \mathrm{mM} \mathrm{Cd}$ pretreated half and control half was discernible. These macroscopic observations suggested that $\mathrm{Cd}$ might bind with some structures of both atrium and ventricle.

\section{Microscopic observation}

Several histochemical techniques ${ }^{5 \sim 7}$ were applied to the sections of the hearts in order to examine the physiological assumption that cadmium binds with cardiac muscle fiber.

The conditions ( $\mathrm{pH}$, concentration of staining solution) for chelating to obtain the largest

\footnotetext{
* Present address : Department of Physiology, Saitama Medical School, Moroyama, Saitama.

** Present address : Department of Physiology, Nippon Medical School, Sendagi, Bunkyo-ku, Tokyo.
} 


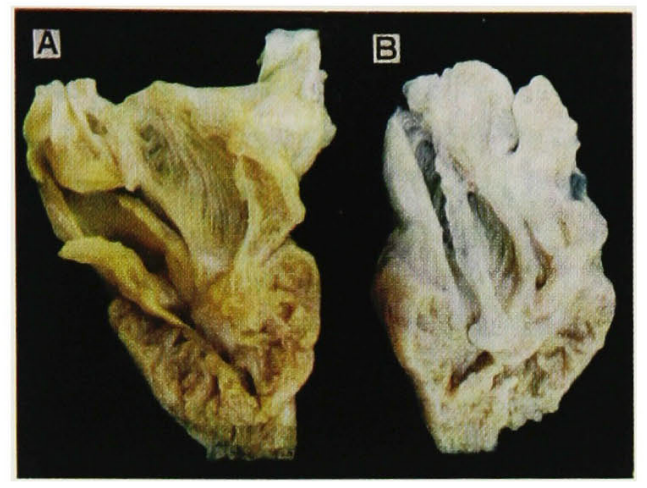

Fig. 1 A divided bull frog heart treated with $\mathrm{H}_{2} \mathrm{~S}$ for 10 minutes.

Left (A): A half of the heart pretreated with $\mathrm{Cd}$ (13 mM 20 minutes). Right (B): A nother controlhalf of the heart without $\mathrm{Cd}$ pretreatment.

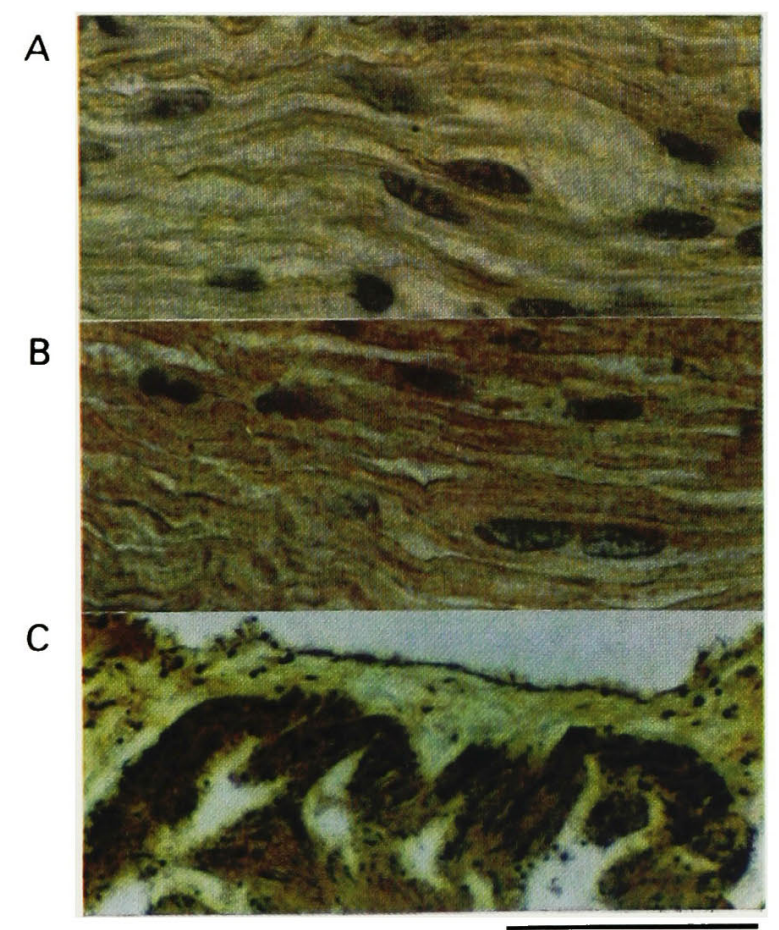

Fig. 2 Photomicrographs of a bull frog atrium. Staining : dithizonehematoxylin.

A : Control (without treatment of $\mathrm{H}_{2} \mathrm{~S}$ ). B : Cd treated $(10 \mathrm{mM}$,60 minutes, without treatment of $\left.\mathrm{H}_{2} \mathrm{~S}\right)$. C : Cd treated $(13 \mathrm{mM}, 20$ minutes, $\mathrm{H}_{2} \mathrm{~S}$ treated before fixation). Calibration : $50 \mu$ for $\mathrm{A}$ aud $\mathrm{B}, 185 \mu$ for $\mathrm{C}$. 
amount of precipitates of cadmium compounds were preliminary determined in test tubes on the following chelating agents : dithizone ${ }^{8)}$, diphenyl thiocarbazide ${ }^{5)}$, xylenol orange, eriochrome black T, methyltymol blue and methylxylenol blue. Among the chelating agents tested, dithizone was found to give the best result to detect cadmium because of its high sensitivity and consistent staining. Okamoto et al. ${ }^{5,6}$ published a dithizone method to detect zinc in pancreas. However, this original technique did not give consistent results for the frog heart. The main points in authors' dithizone staining method are as follows : 1) pH 9.0 dithiz oncRinger's solution (tris buffer $100 \mathrm{mM}, \mathrm{pH} 9.0$ ) instead of unbuffered dithizone ammonium solution of unknown $\mathrm{pH} ; 2)$ mounting dithizone-hematoxylin stained sections with glycerin to avoid effluxion of Cd-dithizone complexes by alcohol which is used in conventional mounting technique.

The hearts were divided into four strips along the heart axes : two of them were soaked in Ringer's solution containing various concentrations of $\mathrm{Cd}$ for 60 minutes and the other two strips were used as the controls. These strips pretreated with or without $\mathrm{Cd}$ were fixed in pure alcohol for 3 days. The $\mathrm{H}_{2} \mathrm{~S}$ treatment before alcohol fixation showed little difference in the results of dithizone staining. The specimens were embedded in paraffin, sectioned in about $8 \mu$ thickness and deparaffinized in a conventional way. Sections were stained in a saturated dithizone-pH 9.0 Ringer's solution for 10 minutes and Mayer's hematoxylin solution for 3 minutes, then after washing in distilled water, they were immediately mounted in glycerin avoiding to use of alcohol before mounting process. Dithizone formed complexes with $\mathrm{Cd}$ in the tissue as fine reddish orange particles. This modified dithizone method greatly increased the sensitivity and it was possible to detect the difference between the controls and $0.01 \mathrm{mM} \mathrm{Cd}$ pretreated hearts. Fig. 2 illustrates the photomicrographs of the sections of bull frog atrium treated with cadmium (B, C) and without cadmium (A). The control (A) never shows fine reddish orange particles of dithizone-Cd complex which are consistently found in the cardiac muscle fiber of cadmium treated hearts (B). It is noteworthy that cadmium distributes not in the connective tissue but almost exclusively in the cardiac muscle fibers (C).

Although the present light microscopic study proved the binding of cadmium to the cardiac muscle fibers, the binding sites of cadmium in relation to the fine structure of cardiac muscle fibers has not yet been elucidated. The results obtained by the electron microscopic study will be reported elsewhere.

REFERENCES

1) Tipton, I.H. : In "Metal binding in medicine”. Lippincott, Philadelphia, 27, 1960.

2) Schroeder, H.A., and Balassa, J.J. : J. Chronic Diseases, 14, 236, 1961.

3) Kobayashi, J. : personal communication

4) Hayashi, H., and Horiuchi, E. : J. Physiol. Soc. Jap., 33, 368, 1971.

5) Okamoto, K., and Hashimoto, N. : Constitutional Med., 13, 83, 1944.

6) Okamoto, K., Ueda, M., and Maeda, R. : In “Microscopic histochemistry”. 100, 1958.

7) Timm, F. : Deutsch. Z. gerichtl. Med., 46, 706, 1958.

8) Fischer, H. : Z. angew. Chem., 46, 442, 1933. 